\section{Response to Biesecker and Harrison}

The American College of Medical Genetics and Genomics/ Association for Molecular Pathology (ACMG/AMP) Interpretation of Sequence Work Group (ISV WG) of 2015 appreciates the opportunity to respond to the recommendations from the ClinGen Sequence Variant Interpretation Working Group. ${ }^{1}$ We commend this expert working group for their thoughtful and sustained efforts to refine the original criteria set forth in the ISV recommendations of $2015 .^{2} \mathrm{We}$ also appreciate and value the many publications that have suggested modifications to the original publication. ${ }^{3-6}$ The ISV WG has not had the opportunity to evaluate thoroughly the consequences of any of these recommended changes on the overall scoring system, including this current one suggesting removal of the supporting-level reputable source criteria (PP5 and BP6), and therefore refrains from making any recommendations at this time.

The ACMG/AMP ISV WG agrees that primary data are preferable to expert opinion. Laboratories may choose to consult ClinVar or other databases to determine how their colleagues have classified a variant, although that in itself would not determine classification. While $81 \%$ of variants reported in ClinVar have evidence provided, there are a significant number of variants (19\%) that do not, and thus could be taken to meet these criteria. Before our committee can evaluate removing a subset of criteria we must determine how removal might impact the current scoring system that utilizes a unified set of criteria for evidence. Thus, it would be important to provide data that show that the use of these two evidence criteria actually leads to errors in variant classification. We assume that removal of the PP5 and BP6 criteria as only supporting evidence is not likely to impact classification (i.e., in the absence of much stronger evidence), although data to support this would be helpful.

Generally, for all ACMG publications, document review is performed every 5 years, unless there is an urgent issue that must be resolved quickly. In that case, a revision to the original document, approved by the participating organizations' Board of Directors (ACMG and AMP in this case), could move forward more quickly to address needed changes. This document was intended to be a complete and comprehensive process for interpretation of genome-level sequence variants for genes published in the literature to be causative of diseases. Therefore, the ISV WG is reluctant to revise individual criteria of the guideline, without careful consideration of the impact on the whole system. Because the field of genetic testing is growing and evolving rapidly, it may be necessary for ACMG to reconvene the ISV WG, which could collaborate directly with the ClinGen Sequence Variant Interpretation Working Group, along with other experts in the field to develop an addendum to, or even revision of, the current guidelines within the next few years. In the meantime, the ISV WG will work with ACMG and AMP to provide additional educational sessions at the annual meetings and perhaps through other venues to update clinical laboratory members on specific areas of the guideline that may require a different approach to variant interpretation. Finally, our work group wholeheartedly agrees with the viewpoint stated in the ClinGen letter that the interpretation of variants is the responsibility of the clinical laboratory director. Therefore, we encourage laboratory directors to seriously consider and evaluate these discussions.

\section{DISCLOSURE}

All work group members are clinical service providers. The following work group members have a commercial conflict of interest: E.S. (Fabric Genomics, consultant); D.B. (Envision Genomics, stock; Genomic England, scientific advisor); K.V. (Pierian Dx, scientific advisor); E.L. (Complete Genomics, advisory board; Roche, advisory board; Genome Canada, reviewer). The other authors declare no conflict of interest.

C. Sue Richards, $P h D^{1}$, Nazneen Aziz, $P h D^{2}$, Sherri Bale, $P h D^{3}$, David Bick, $M D^{4}$, Soma Das, $P h D^{5}$, Julie Gastier-Foster, $P h D^{6}$, Wayne W. Grody, MD, $P h D^{7}$, Madhuri Hegde, $P h D^{8}$, Elaine Lyon, $P h D^{9}$, Elaine Spector, $P h D^{10}$, Karl Voelkerding, $M D^{11}$, Heidi L. Rehm, $P h D^{12}$ and on behalf of the ACMG/AMP Interpretation of Sequence Variants Work Group 2015

${ }^{1}$ Knight Diagnostic Laboratories, Department of Molecular and Medical Genetics, Oregon Health \& Science University, Portland, Oregon, USA; ${ }^{2}$ Kaiser Permanente, Oakland, California, USA; ${ }^{3}$ GeneDX, Gaithersburg, Maryland, USA; ${ }^{4}$ HudsonAlpha Institute for Biotechnology, Huntsville, Alabama, USA; ${ }^{5}$ Department of Human Genetics, Clinical Molecular Genetics Laboratory, The University of Chicago, Chicago, Illinois, USA; ${ }^{6}$ Institute for Genomic Medicine Clinical Laboratory, Nationwide Children's Hospital, The Ohio State University College of Medicine, Columbus, Ohio, USA; ${ }^{7}$ Molecular Diagnostic Laboratories and Clinical Genomics Center, UCLA School of Medicine, Los Angeles, California, USA; ${ }^{8}$ Global Laboratory Services/Diagnostics, Perkin Elmer, Waltham, Massachusetts, USA; ${ }^{9}$ Department of Pathology, ARUP Institute for Clinical and Experimental Pathology, University of Utah, Salt Lake City, Utah, USA; ${ }^{10}$ Department of Pediatrics, Molecular Genetics Laboratory, Children's Hospital Colorado, University of Colorado Medical School, Denver, Colorado, USA;

${ }^{11}$ University of Utah Department of Pathology, ARUP Laboratories, Salt Lake City,

Utah, USA; ${ }^{12}$ Partners Laboratory for Molecular Medicine and Department of

Pathology, Brigham \& Women's Hospital and Harvard Medical School, Boston,

Massachusetts, USA. Correspondence: Sue Richards (richarsu@ohsu.edu)

\section{REFERENCES}

1. Biesecker LG \& Harrison SM. The ACMG/AMP reputable source criteria for the interpretation of sequence variants. Genet Med 2018;20: $\mathrm{XXX}-\mathrm{XXX}$.

2. Richards S, Aziz N, Bale E, et al. Standards and guidelines for the interpretation of sequence variants: a joint consensus recommendation of the American College of Medical Genetics and Genomics and the Association for Molecular Pathology. Genet Med 2015;17:405-424. 


\section{LETTER TO THE EDITOR}

3. Kleinberger J, Maloney KA, Pollin TI \& Jeng L. An openly available online tool for implementing the ACMG/AMP standards and guidelines for the interpretation of sequence variants. Genet Med 2016;18:1165.

4. Nykamp K, Anderson M, Powers M, et al. Sherloc: a comprehensive refinement of the ACMG-AMP variant classification criteria. Genet Med 2017;19:1105-1117.

5. Kelly MA, Caleshu C, Morales A, et al. Adaptation and validation of the ACMG/AMP variant classification framework for MYH7-associated
Letter to the Editor | Response to Biesecker and Harrison

inherited cardiomyopathies: recommendations by ClinGen's Inherited Cardiomyopathy Expert Panel. Genet Med 2018;20:351-359.

6. Jarvik GP \& Browning BL. Consideration of cosegregation in the pathogenicity classification of genomic variants. Am J Hum Genet 2016;98:1077-1081.

Advance online publication 15 March 2018. doi:10.1038/gim.2018.43 